Turk. J. Math. Comput. Sci.

13(2)(2021) 373-378

(C) MatDer

DOI : $10.47000 /$ tjmcs.911049

\title{
Spectral Properties of the Sturm-Liouville Operator Produced by the Unseparated Boundary Conditions with Spectral Parameter
}

\author{
Rauf Amirov $^{1}$ (D), Selma Gülyaz Özyurt ${ }^{1, *}$ (D) \\ ${ }^{1}$ Department of Mathematics, Faculty of Science, Sivas Cumhuriyet University, 58140 Sivas, Turkey.
}

Received: 07-04-2021 • Accepted: 03-07-2021

\begin{abstract}
In this study, firstly, the basic properties of the spectrum of the investigated problem were learned, sine and cosine type solutions were defined, their behaviors were examined and the properties of the solution of the given problem were learned with their help. Next, the characteristic equation of the studied problem was formed with the help of sine and cosine type solutions. Using the characteristic equation, the asymptotic behavior of the eigenvalues of the given problem and the ordering of the eigenvalues of the boundary value problems $L\left(\alpha_{j}\right), j=1$, 2 when $\alpha_{1}<\alpha_{2}$ are learned.
\end{abstract}

2010 AMS Classification: 34A55, 34B24, 34L05

Keywords: Eigenvalue, eigenfunction, unseparated boundary conditions.

\section{INTRODUCTION}

Let us consider the boundary value problem $L(\alpha)$ :

$$
\begin{gathered}
-y^{\prime \prime}+q(x) y=\lambda y, 0<x<\pi, \lambda=\rho^{2} \\
y^{\prime}(0)+(\alpha \lambda+\beta) y(0)+\omega y(\pi)=0, \\
y^{\prime}(\pi)+\gamma y(\pi)-\bar{\omega} y(0)=0,
\end{gathered}
$$

where $\alpha, \beta, \gamma$ are real numbers, $\omega$ is a complex number and such that $\alpha \omega \neq 0, q(x)$ is a real-valued function in $L_{2}[0, \pi]$.

The solution to many problems of applied sciences and mathematical physics is reduced to the problem of examining (1.1) - (1.2) boundary value problems.

When $\omega=0,(1.2)$ the boundary conditions become separated boundary conditions depending on the spectral parameter. Spectral properties of operators produced by Sturm-Liouville and diffusion equations given with separated boundary conditions depending on the spectral parameter were learned in $[1,5,6,10,15]$ studies. In the studies of $[2-4,7-9,11-14,16]]$, straight and inverse problems were investigated for diffusion equations given with different unseparated boundary conditions at $p(x) \equiv 0$ and $p(x) \neq 0$.

In this study, important properties of the spectrum of the $L(\alpha)$ boundary value problem was learned when $\alpha \neq 0$, $\omega \neq 0$. In the case of one of the unseparated boundary conditions is linearly dependent on the spectral parameter, the basic properties of the spectrum of the $L(\alpha)$ boundary value problem are given. In our study, the eigenvalues of the boundary value problem $L(\alpha)$ are real, the absence of conditions that function, the asymptotic behavior of the

*Corresponding Author

Email addresses: emirov@cumhuriyet.edu.tr (R. Amirov), sgulyaz@cumhuriyet.edu.tr (S. Gülyaz Özyurt) 
eigenvalues and finally the ordering of the eigenvalues of the boundary value problems $L\left(\alpha_{1}\right)$ and $L\left(\alpha_{2}\right)$ when $\alpha_{1}<\alpha_{2}$ is examined.

\section{Main Results}

In this section, the eigenvalues of the $L(\alpha)$ boundary value problem and the properties of the eigenfunctions will be learned and the asymptotic behavior of the eigenvalues will be obtained.

$C(x, \lambda)$ and $S(x, \lambda)$, are linearly independent of equation (1.1) and

$$
C(0, \lambda)=S^{\prime}(0, \lambda)=1, \quad C^{\prime}(0, \lambda)=S(0, \lambda)=0
$$

get solutions that meet the initial conditions.

To obtain the main results of the study, let's first give the Lemma as follow.

Lemma 2.1. The following representations are valid:

$$
\begin{aligned}
& C(\pi, \lambda)=\cos \rho \pi+\pi c_{0} \frac{\sin \rho \pi}{\rho}+\frac{1}{\rho} \int_{-\pi}^{\pi} \psi_{1}(t) e^{i \rho t} d t \\
& C^{\prime}(\pi, \lambda)=-\rho \sin \rho \pi+\pi c_{0} \cos \rho \pi+\frac{1}{\rho} \int_{-\pi}^{\pi} \psi_{2}(t) e^{i \rho t} d t \\
& S(\pi, \lambda)=\frac{\sin \rho \pi}{\rho}-\pi c_{0} \frac{\cos \rho \pi}{\rho^{2}}+\frac{1}{\rho^{2}} \int_{-\pi}^{\pi} \psi_{3}(t) e^{i \rho t} d t \\
& S^{\prime}(\pi, \lambda)=\cos \rho \pi+\pi c_{0} \frac{\sin \rho \pi}{\rho}+\frac{1}{\rho} \int_{-\pi}^{\pi} \psi_{4}(t) e^{i \rho t} d t
\end{aligned}
$$

where $c_{0}=\frac{1}{2 \pi} \int_{0}^{\pi} q(t) d t, \psi_{i}(t) \in L_{2}[-\pi, \pi], i=\overline{1,4}$.

The proof of Lemma 2.1 is done similarly to the proof of Lemma 1.3.2 in [ [15]].

Definition 2.2. A complex number $\lambda_{0}$ is called an eigenvalue of boundary value problem $L(\alpha)$ if equation (1.1) with $\lambda=\lambda_{0}$ has a nontrivial solution $y_{0}(x)$ is called the eigenfunction of problem $L(\alpha)$ corresponding to the eigenvalue $\lambda_{0}$. The set of eigenvalues is called the spectrum of $L(\alpha)$. The functions $y_{1}(x), y_{2}(x), \ldots, y_{k}(x)$ are called as the associated functions to the eigenfunction $y_{0}(x)$, if they absolutely continuous, then they satisfy the differential equations

$$
-y_{i}^{\prime \prime}(x)+q(x) y_{i}(x)-y_{i-1}(x)=\lambda_{0} y_{i}(x)
$$

and boundary conditions

$$
\begin{gathered}
y_{i}^{\prime}(0)+(\alpha \lambda+\beta) y_{i}(0)+\alpha y_{i-1}(0)+\omega y_{i}(\pi)=0, \\
y_{i}^{\prime}(\pi)+\gamma y_{i}(\pi)-\varpi y_{i}(0)=0, \quad i=\overline{1, k} .
\end{gathered}
$$

Theorem 2.3. If $\alpha<0$, then the eigenvalues of the problem $L(\alpha)$ are real.

Proof. Let's assume the opposite, i.e. $\operatorname{Im} \lambda \neq 0$. Let $\lambda$ be an eigenvalue of the boundary problem $L(\alpha)$, and let $y(x)=$ $y(x, \lambda)$ be the corresponding eigenfunction. Multiply the equality (1.1) by $\overline{y(x)}$,

$$
-\overline{y^{\prime \prime}(x)}+q(x) \overline{y(x)}=\lambda \overline{y(x)}
$$

by $y(x)$ and subtract one product from the other. As a result, we get

$$
(\lambda-\bar{\lambda})|y(x)|^{2}=\frac{d}{d x}\left[\overline{y(x)} y^{\prime}(x)-y(x) \overline{y^{\prime}(x)}\right] .
$$

Integrating this equality from zero to $\pi$, then we have

$$
2 i \operatorname{Im} \lambda \int_{0}^{\pi}|y(x)|^{2} d x=\overline{y(\pi)} y^{\prime}(\pi)-y(\pi) \overline{y^{\prime}(\pi)}-\overline{y(0)} y^{\prime}(0)+y(0) \overline{y^{\prime}(0)} \text {. }
$$


It follows from the boundary conditions (1.2) there are

$$
\begin{aligned}
& y^{\prime}(0)=-(\alpha \lambda+\beta) y(0)-\omega y(\pi), \\
& y^{\prime}(\pi)=-\gamma y(\pi)+\varpi y(0) .
\end{aligned}
$$

Let's consider these at the equality (2.3)

$$
\begin{aligned}
2 i \operatorname{Im} \lambda \int_{0}^{\pi}|y(x)|^{2} d x= & \overline{y(\pi)}[-\gamma y(\pi)+\varpi y(0)]-y(\pi)[-\gamma \overline{y(\pi)}+\omega \overline{y(0)}] \\
& -\overline{y(0)}[-(\alpha \lambda+\beta) y(0)-\omega y(\pi)]+y(0)[-(\alpha \bar{\lambda}+\beta) \overline{y(0)}-\bar{\omega} \overline{y(\pi)}] \\
= & 2 i \alpha \operatorname{Im} \lambda|y(0)|^{2} .
\end{aligned}
$$

Consequently,

$$
\operatorname{Im} \lambda\left\{\int_{0}^{\pi}|y(x)|^{2} d x-\alpha|y(0)|^{2}\right\}=0 .
$$

Since $\alpha<0$, the expression in braces is distinct from zero. Therefore, $\operatorname{Im} \lambda=0$. Theorem is proved.

Theorem 2.4. If $\alpha<0$, then the eigenfunctions of the boundary value problem $L(\alpha)$ have no associated functions.

Proof. Let's assume the opposite. Suppose that the boundary value problem $L(\alpha)$ has an eigenfunction of $y_{1}(x)$ associated to the $y_{0}(x)$ eigenfunction, corresponding to the eigenvalue $\lambda_{0}$. Then, by virtue of (1.1) and (2.2), the following equalities hold:

$$
\begin{gathered}
-\overline{y_{0}^{\prime \prime}(x)}+q(x) \overline{y_{0}(x)}=\lambda_{0} \overline{y_{0}(x)} \\
-y_{1}^{\prime \prime}(x)+q(x) y_{1}(x)-y_{0}(x)=\lambda_{0} y_{1}(x) .
\end{gathered}
$$

Multiply the equality (2.4) by $y_{1}(x)$ and equality (2.5) by $\overline{y_{0}(x)}$ and subtract them side by side, we will get

$$
\left|y_{0}(x)\right|^{2}=\frac{d}{d x}\left[y_{1}^{\prime}(x) \overline{y_{0}(x)}-y_{1}(x) \overline{y_{0}^{\prime}(x)}\right] .
$$

Integrating this equality from zero to $\pi$ for $x$, we obtain

$$
\int_{0}^{\pi}\left|y_{0}(x)\right|^{2} d x=y_{1}^{\prime}(\pi) \overline{y_{0}(\pi)}-y_{1}(\pi) \overline{y_{0}^{\prime}(\pi)}-y_{1}^{\prime}(0) \overline{y_{0}(0)}+y_{1}(0) \overline{y_{0}^{\prime}(0)} .
$$

According to the boundary conditions (1.2) and (2.2), we have

$$
\begin{aligned}
& y_{0}^{\prime}(0)=-(\alpha \lambda+\beta) y_{0}(0)-\omega y_{0}(\pi), \\
& y_{0}^{\prime}(\pi)=-\gamma y_{0}(\pi)+\varpi y_{0}(0), \\
& y_{1}^{\prime}(0)=-(\alpha \lambda+\beta) y_{1}(0)-\omega y_{1}(\pi)-\alpha y_{0}(0), \\
& y_{1}^{\prime}(\pi)=-\gamma y_{1}(\pi)+\varpi y_{1}(0) . \\
& \int_{0}^{\pi}\left|y_{0}(x)\right|^{2} d x=\overline{y_{0}(\pi)}\left[-\gamma y_{1}(\pi)+\varpi y_{1}(0)\right]-y_{1}(\pi)\left[-\gamma \overline{y_{0}(\pi)}+\omega \overline{y_{0}(0)}\right] \\
&=\alpha \overline{y_{0}(0)}\left[(\alpha \lambda+\beta) y_{1}(0)-\omega y_{1}(\pi)+\alpha y_{0}(0)\right]-y_{1}(0)\left[(\alpha \bar{\lambda}+\beta) \overline{y_{0}(0)}+\bar{\omega} \overline{y_{0}(\pi)}\right]
\end{aligned}
$$


Hence

$$
\int_{0}^{\pi}\left|y_{0}(x)\right|^{2} d x-\alpha\left|y_{0}(0)\right|^{2}=0 .
$$

This contradicts the fact that the left side of this relation is pozitive according to the inequality $\alpha<0$. Theorem is proved.

The general solution of the equation (1.1) has a form

$$
y(x, \lambda)=A S(x, \lambda)+B C(x, \lambda)
$$

where $A$ and $B$ are arbitrary constant. Considering the boundary conditions (1.2) and initial conditions (2.1) taking into account the identity

$$
C(x, \lambda)\left[S^{\prime}(x, \lambda)+\gamma S(x, \lambda)\right]-S(x, \lambda)\left[C^{\prime}(x, \lambda)+\gamma C(x, \lambda)\right]=1,
$$

it is easy to verify that the characteristic function of the boundary value problem $L(\alpha)$ will be

$$
\begin{gathered}
\Delta(\lambda)=2 \operatorname{Re} \omega-\left[C^{\prime}(\pi, \lambda)+\gamma C(\pi, \lambda)\right]+|\omega|^{2} S(\pi, \lambda) \\
+(\alpha \lambda+\beta)\left[S^{\prime}(\pi, \lambda)+\gamma S(\pi, \lambda)\right] .
\end{gathered}
$$

The square of zeros of the characteristic function $\Delta(\lambda)$ are the eigenvalues of the problem $L(\alpha)$.

Using the representation in the Lemma 1, we transform the characteristicfunction (2.6) to the form

$$
\begin{aligned}
\Delta(\lambda) & =\alpha \rho^{2} \cos \rho \pi+\left(1+\alpha \pi c_{0}\right) \rho \sin \rho \pi+\alpha \gamma \sin \rho \pi \\
& +\left(\beta-\alpha \gamma \pi c_{0-} \pi c_{0}-\gamma\right) \cos \rho \pi+2 \operatorname{Re} \omega+\psi_{\xi}(\lambda)
\end{aligned}
$$

where $\psi_{\xi}(\lambda)=\int_{-\pi}^{\pi} \widetilde{\psi}_{\xi}(t) e^{i \lambda t} d t, \quad \widetilde{\psi}_{\xi}(t) \in L_{2}[-\pi, \pi]$.

Let $\Delta_{0}(\lambda)=\alpha \rho^{2} \cos \rho \pi+\rho \sin \rho \pi+\alpha \gamma \sin \rho \pi+(\beta-\gamma) \cos \rho \pi+2 \operatorname{Re} \omega$.

Using Lemma 1 which was proven in study [7], we can easily prove the following proposition.

Theorem 2.5. The following statements hold.

a) Let $\lambda_{n}$ be the $n$th eigenvalue of the problem (1.1)-(1.2). Let $\left\{\mu_{k}\right\}_{k \geq 0}$ be the zeros of the entire function $\Delta_{0}(\lambda)$. If $\mu_{p}$ is the closest point to $\lambda_{n}$ then as $n \rightarrow \infty$

$$
\rho_{n}=\sqrt{\mu_{p}}+O\left(\frac{1}{\sqrt{n}}\right) .
$$

In addition, if there exists a positive number $\sigma_{0}$ such that $\left\{\mu_{k}\right\}_{k \geq 0}$ satisfy

$$
\left|\sqrt{\mu_{k}}-\sqrt{\mu_{k^{\prime}}}\right|>\sigma_{0} \text { as } \mu_{k} \neq \mu_{k^{\prime}}
$$

then (2.7) holds for $n=p$.

b) Fix $\delta>0$. Then there exists a constants $C_{\delta}$ such that

$$
|\Delta(\lambda)|>C_{\delta}|\rho|^{2}, \rho \in G_{\delta},|\rho| \geq \rho^{*}
$$

for sufficiently large $\rho^{*}$, where $G_{\delta}=\left\{\rho\left|\rho-\sqrt{\mu_{n}}\right| \geq \delta, n \geq 0\right\}$.

It should be noted that $\left\{\mu_{n}\right\}_{n \geq 0}$ are independent of the potential $q(x)$.

Furthermore, it is easy to see that

$$
\lim _{n \rightarrow \infty} \frac{\sqrt{\mu_{p}}}{n}=a_{1}, \lim _{n \rightarrow \infty} \frac{\rho_{n}}{\sqrt{\mu_{p}}}=a_{2},
$$

where both $a_{1}$ and $a_{2}$ are positive constans.

For the $j=1,2$ denote the characteristic functions of the boundary value problems $L\left(\alpha_{j}\right)$ by

$$
\begin{aligned}
\Delta_{j}(\lambda) & =2 \operatorname{Re} \omega-\left[C^{\prime}(\pi, \lambda)+\gamma C(\pi, \lambda)\right]+|\omega|^{2} S(\pi, \lambda) \\
& +\left(\alpha_{j} \lambda+\beta\right)\left[S^{\prime}(\pi, \lambda)+\gamma S(\pi, \lambda)\right]
\end{aligned}
$$


and the negative eigenvalues arranged in increasing order by

$$
\lambda_{0}^{-}(j), \lambda_{-1}^{+}(j), \lambda_{-1}^{-}(j), \lambda_{-2}^{+}(j), \lambda_{-2}^{-}(j), \ldots
$$

the positive eigenvalues arranged in increasing order by

$$
\lambda_{0}^{+}(j), \lambda_{1}^{-}(j), \lambda_{1}^{+}(j), \lambda_{2}^{-}(j), \lambda_{2}^{+}(j), \ldots
$$

Theorem 2.6. The relative pozition of the eigenvalues $\lambda_{k}^{ \pm}(1)$ and $\lambda_{k}^{ \pm}(2)(k=0, \pm 1, \pm 2, \ldots)$ of the boundary value problems $L\left(\alpha_{1}\right)$ and $L\left(\alpha_{2}\right)$ with $\alpha_{1}<\alpha_{2}$ is determined for Im $\omega \neq 0$ by the inequalities

$0<\lambda_{0}^{+}(1)<\lambda_{0}^{+}(2)<\lambda_{1}^{-}(1)<\lambda_{1}^{-}(2)<\lambda_{1}^{+}(1)<\lambda_{1}^{+}(2)<\lambda_{2}^{-}(1)<\lambda_{2}^{-}(2)<\ldots$,

$0>\lambda_{0}^{-}(1)>\lambda_{0}^{-}(2)>\lambda_{-1}^{+}(1)>\lambda_{-1}^{+}(2)>\lambda_{-1}^{-}(1)>\lambda_{-1}^{-}(2)>\lambda_{-2}^{+}(1)>\lambda_{-2}^{+}(2)>\ldots$,

for real $\omega<0$ by the inequalities

$0<\lambda_{0}^{+}(1)<\lambda_{0}^{+}(2)<\lambda_{1}^{-}(1)<\lambda_{1}^{-}(2)<\lambda_{1}^{+}(1) \leq \lambda_{1}^{+}(2)<\lambda_{2}^{-}(1) \leq \lambda_{2}^{-}(2) \leq \ldots$,

$0>\lambda_{0}^{-}(1)>\lambda_{0}^{-}(2)>\lambda_{-1}^{+}(1)>\lambda_{-1}^{+}(2) \geq \lambda_{-1}^{-}(1) \geq \lambda_{-1}^{-}(2)>\lambda_{-2}^{+}(1) \geq \lambda_{-2}^{+}(2) \geq \ldots$,

and for real $\omega>0$ by the inequalities

$0<\lambda_{0}^{+}(1) \leq \lambda_{0}^{+}(2) \leq \lambda_{1}^{-}(1) \leq \lambda_{1}^{-}(2)<\lambda_{1}^{+}(1) \leq \lambda_{1}^{+}(2) \leq \lambda_{2}^{-}(1) \leq \lambda_{2}^{-}(2)<\ldots$,

$0>\lambda_{0}^{-}(1) \geq \lambda_{0}^{-}(2) \geq \lambda_{-1}^{+}(1) \geq \lambda_{-1}^{+}(2)>\lambda_{-1}^{-}(1) \geq \lambda_{-1}^{-}(2) \geq \lambda_{-2}^{+}(1) \geq \lambda_{-2}^{+}(2)>\ldots$

Proof. It is clear that function

$$
U(x, \lambda)=\left[S^{\prime}(\pi, \lambda)+\gamma S(\pi, \lambda)\right] C(x, \lambda)+\left[\omega-C^{\prime}(\pi, \lambda)-\gamma C(\pi, \lambda)\right] S(x, \lambda)
$$

is the solution of the equation (1.1) that satisfies the initial conditions

$$
U(0, \lambda)=S^{\prime}(\pi, \lambda)+\gamma S(\pi, \lambda), U^{\prime}(0, \lambda)=\omega-C^{\prime}(\pi, \lambda)-\gamma C(\pi, \lambda) .
$$

Also,

$$
U(\pi, \lambda)=\omega S(\pi, \lambda)+1, U^{\prime}(0 \pi, \lambda)=\omega S^{\prime}(\pi, \lambda)-\gamma
$$

conditions are met at the point $x=\pi$.

By differentiating the equation

$$
U^{\prime \prime}(x, \lambda)+(\lambda-q(x)) U(x, \lambda)=0
$$

with respect to $\lambda$ and by passing to the complex conjugate in the resulting equation, for reel $\lambda$ we obtain

$$
\overline{\dot{U}^{\prime \prime}(x, \lambda)}+(\lambda-q(x) \overline{\bar{U}(x, \lambda)}+\overline{U(x, \lambda)}=0 .
$$

Next, we multiply this equation by $U(x, \lambda)$ and equation $(2.11)$ by $\dot{U}(x, \lambda)$, subtract the second result from the first, and integrate with respect to $x$ over $[0, \pi]$; by (2.9) and (2.10) and since the functions $C(\pi, \lambda), C^{\prime}(\pi, \lambda), S(\pi, \lambda)$ and $S^{\prime}(\pi, \lambda)$ are real, we obtain

$$
\begin{aligned}
& \int_{0}^{\pi}|U(x, \lambda)|^{2} d x=\overline{\dot{U}(\pi, \lambda)} U^{\prime}(\pi, \lambda)-\overline{\dot{U}^{\prime}(\pi, \lambda)} U(\pi, \lambda)-\overline{\dot{U}(0, \lambda)} U^{\prime}(0, \lambda)+\overline{\dot{U}^{\prime}(0, \lambda)} U(0, \lambda) \\
& =\left[\omega S^{\prime}(\pi, \lambda)-\gamma\right] \bar{\omega} \dot{S}(\pi, \lambda)-\bar{\omega} \dot{S}^{\prime}(\pi, \lambda)[\omega S(\pi, \lambda)+1]-\left[\omega-C^{\prime}(\pi, \lambda)-\gamma C(\pi, \lambda)\right] \\
& \times\left[\dot{S^{\prime}}(\pi, \lambda)+\gamma \dot{S}(\pi, \lambda)\right]-\left[\dot{C^{\prime}}(\pi, \lambda)+\gamma \dot{C}(\pi, \lambda)\right]\left[S^{\prime}(\pi, \lambda)+\gamma S(\pi, \lambda)\right] \\
& =|\omega|^{2} S^{\prime}(\pi, \lambda) \dot{S}(\pi, \lambda)-\gamma \bar{\omega} \dot{S}(\pi, \lambda)+|\omega|^{2} S(\pi, \lambda) \dot{S}(\pi, \lambda)-\bar{\omega} \dot{S}^{\prime}(\pi, \lambda) \\
& -\omega\left[\dot{S^{\prime}}(\pi, \lambda)+\gamma \dot{S}(\pi, \lambda)\right]+\left[C^{\prime}(\pi, \lambda)+\gamma C(\pi, \lambda)\right]\left[\dot{S^{\prime}}(\pi, \lambda)+\gamma \dot{S}(\pi, \lambda)\right] \\
& -\left[\dot{C}^{\prime}(\pi, \lambda)+\gamma \dot{C}(\pi, \lambda)\right]\left[S^{\prime}(\pi, \lambda)+\gamma S(\pi, \lambda)\right] \\
& =-\left[\dot{S^{\prime}}(\pi, \lambda)+\gamma \dot{S}(\pi, \lambda)\right]\left[2 \operatorname{Re} \omega-C^{\prime}(\pi, \lambda)-\gamma C(\pi, \lambda)+|\omega|^{2} S(\pi, \lambda)+\beta\left(S^{\prime}(\pi, \lambda)+\gamma S(\pi, \lambda)\right)\right] \\
& +\left[S^{\prime}(\pi, \lambda)+\gamma S(\pi, \lambda)\right]\left[|\omega|^{2} \dot{S}(\pi, \lambda)-\dot{C}^{\prime}(\pi, \lambda)-\gamma \dot{C}(\pi, \lambda)+\beta\left(\dot{S^{\prime}}(\pi, \lambda)+\gamma \dot{S}(\pi, \lambda)\right)\right] .
\end{aligned}
$$


With regard for the equations

$$
\begin{gathered}
S^{\prime}(\pi, \lambda)+\gamma S(\pi, \lambda)=\frac{\Delta_{1}(\lambda)-\Delta_{2}(\lambda)}{\left(\alpha_{1}-\alpha_{2}\right) \lambda}, \\
2 \operatorname{Re} \omega-\left[C^{\prime}(\pi, \lambda)+\gamma C(\pi, \lambda)\right]+\beta\left[S^{\prime}(\pi, \lambda)+\gamma S(\pi, \lambda)\right]+|\omega|^{2} S(\pi, \lambda)=\frac{\alpha_{2} \Delta_{1}(\lambda)-\alpha_{1} \Delta_{2}(\lambda)}{\alpha_{2}-\alpha_{1}},
\end{gathered}
$$

which follows from (2.8), we find that

$$
\left(\alpha_{2}-\alpha_{1}\right)\left\{\int_{0}^{\pi}|U(x, \lambda)|^{2} d x+\alpha_{j}|U(0, \lambda)|^{2}\right\}=\frac{\dot{\Delta}_{1}(\lambda) \Delta_{2}(\lambda)-\Delta_{1}(\lambda) \dot{\Delta}_{2}(\lambda)}{\lambda}+\frac{\left[\Delta_{2}(\lambda)-\Delta_{1}(\lambda)\right] \Delta_{j}(\lambda)}{\lambda^{2}}, \quad(j=1,2)
$$

or

$$
\lambda^{2}\left(\alpha_{2}-\alpha_{1}\right)\left\{\int_{0}^{\pi}|U(x, \lambda)|^{2} d x+\alpha_{j}|U(0, \lambda)|^{2}\right\}=\lambda\left[\Delta_{2}(\lambda)\right]^{2} \frac{d}{d \lambda}\left[\frac{\Delta_{1}(\lambda)}{\Delta_{2}(\lambda)}\right]+\left[\Delta_{2}(\lambda)-\Delta_{1}(\lambda)\right] \Delta_{j}(\lambda), \quad(j=1,2) .
$$

If we make use of [12], we get the inequalities in the ruling of the theorem.

\section{CONFLICTS OF INTEREST}

The authors declare that there no conflicts of interest regarding the publication of this paper.

\section{Authors Contribution Statement}

The authors contributed equally to this study. All authors have read and agreed to the published version of the manuscript.

\section{REFERENCES}

[1] Amirov, R.Kh., Cakmak, Y., Inverse problem for Sturm-Liouville operator with respect to a spectrum and normalizing numbers, Cumhuriyet Journal of Science, 24(1)(2003), 34-50.

[2] Amirov, R.Kh., Keskin, B., Ozkan, A.S., Direct and inverse problems for the impulsive Sturm-Liouville boundary value problem where boundary conditions include the spectral parameter, Cumhuriyet Journal of Science, 27(2)(2006), 13-23.

[3] Amirov, R., Durak, S., Behaviors of eigenvalues and eigenfunctions of the singular Shrödinger operator, Turkish Journal of Mathematics and Computer Science, 12(2)(2020), 151-156.

[4] Gasymov, M.G., Guseinov, I.M., Nabiev, I.M., An inverse problem for the Sturm-Liouville operator with nonseparable self-adjoint boundary conditions, Siberian Mathematical Journal, 31(6)(1990), 910-918.

[5] Gasymov, M.G., Guseinov, G. Sh., Reconstruction of a diffusion operator from the spectral data, Dokl. Akad.Nauk. Azerb. SSR, 37(2)(1981), $19-23$.

[6] Guliyev, N.J., Inverse eigenvalue problems for Sturm-Liouville equations with spectral parameter linearly contained in one of the boundary conditions, Inverse Problems, IOP Publishing, 21(4)(2005), 1315-1330.

[7] Guo, Y., Wei, G., On the reconstruction of the Sturm-Liouville problems with spectral parameter in the discontinuity conditions, Results. Math., 65(2014), 385-398.

[8] Guseinov, I.M., Nabiev, I.M., On one class of inverse boundary-value problems for the Strum-Liouville operators, Differents. Uravn., 25(7)(1989), 1114-1120.

[9] Guseinov, I.M., Nabiev, I.M., Solution of a class of inverse boundary-value Sturm-Liouville problems, Sbornik: Mathematics, 186(5)(1995), $661-674$.

[10] Güldü, Y., Amirov, R.K., Topsakal, N., On impulsive Sturm-Liouville operators with singularity and spectral parameter in boundary conditions, Ukrainian Mathematical Journal, 64(2013), 1816-1838.

[11] Ibadzadeh, Ch.G., Nabiyev, I.M., An inverse problem for Sturm-Liouville operator with non-separated boundary conditions containing the spectral parameter, Journal of Inverse and III Posed Problems, 24(4)(2016), 407-411.

[12] Nabiev, I.M., Multiplicities and relative position of eigenvalues of a quadratic pencil of Sturm-Liouville operators, Mathematical Notes, 67(3)(2000), 309-319.

[13] Nabiev, I.M., The uniqueness of reconstruction of quadratic bundle for Sturm-Liouville operators, Proceedings of IMM of NAS of Azerbaijan, 23(2004), 91-96.

[14] Nabiev, I.M., Shukurov, A.Sh., Properties of the spectrum and uniqueness of reconstruction of Sturm-Liouville operator with a spectral parameter in the boundary condition, Proc. of the Institute of Mathematics and Mechanics, National Academy of Sciences of Azerbaijan, 40, Special Issue, (2014), 332-341.

[15] Marchenko, V.A., Sturm-Liouville Operators and Their Applications, Naukova Dumka, Kiev, 1977, English transl.: Birkhäuser, 1986.

[16] Yurko, V.A., The inverse spectral problem for differential operators with non-separated boundary conditions, Journal of Inverse and III Posed Problems, 28(4)(2020), 567-616. 